\title{
An Update of Ovulation Induction
}

\author{
Jamil A Fayez, M.D., Sulieman A. Al-Sulieman, M.D.* \\ Winston-Salem, North Carolina
}

DOI: http://dx.doi.org/10.5915/20-2-13270

\begin{abstract}
In the past decade, some of the most dramatic advances in obstetrics and gynecology have been in ovulation induction. This article is a step-by-step guide to the diagnostic etiology and management of anovulation. The physiologic and pharmacologic functions of the currently marketed ovulatory agents are described.
\end{abstract}

Key Words: Ovulation induction, ovulatory agents, oligomenorrhea, amenorrhea, galactorrhea, polycystic ovaries, luteal phase defect

Lack of ovulation is the primary defect in $25 \%$ of infertile couples and may present as oligomenorrhea or amenorrhea. The oligomenorrheic patient possesses gonadotropins and estrogen but does not ovulate cyclically with any predicatable frequency because her periods vary between 1 to 4 months in frequency. Most amenorrheic patients are usually totally deficient in gonadotropins and therefore do not produce estrogen. Amenorrheic patients with ovarian failure have high levels of gonadotropins and these patients are obviously not candidates for ovulation induction.

\section{Workup prior to therapy}

Before beginning ovulation induction, patients should be assessed for contraindications to therapy. Complete history and an adequate physical examination are mandatory so that no reversible disease is overlooked and that the nature of the anovulation is understood. Inquire about the frequency of menstrual cycles, history of psychological trauma, occurrence of hot flashes, previous oral contraceptive intake and their type, presence of hirsutism or

From the Department of Obstetrics and Gynecology, Section of Reproductive Endocrinology

Bowman Gray School of Medicine

of Wake Forest University

Winston-Salem, North Carolina 27103

*Present Address:

Medical College of King Faisal University

Dammam, Saudi Arabia

Reprint Requests: Jamil A. Fayez, M.D.

Section of Reproductive Endocrinology

Bowman Gray School of Medicine

300 South Hawthorne Road

Winston-Salem, North Carolina 27103 milk secretion from one or both breasts. The physical examination should rule out or confirm the presence of hirsutism or galactorrhea. The pelvic examination should rule out the existence of a pelvic mass such as an ovarian tumor that may be responsible for anovulation. Laboratory tests may include blood levels of follicle stimulating hormone (FSH) and luteinizing hormone ( $\mathrm{LH})$ to rule out premature ovarian failure; measurement of prolactin (PRL) to rule out or confirm hyperprolactinemia, and thyroid stimulating hormone (TSH) which may uncover a subtle case of hypothyroidism. All of these laboratory tests may be required depending on the judgment and experience of the treating physician (Table 1). Semen analysis and hysterosalpingogram are mandatory for obvious reasons.

\section{Selection of patients}

The usual therapeutic approach to the induction of ovulation has beeb based on patients' signs and symptoms such as amenorrhea, oligomenorrhea and dysfunctional uterine bleeding without dealing with the problem from the diagnostic point of view. The diagnostic approach is not only more meaningful to clinicians but it also solves the dilemma of what is the best ovulating agent for each potential recipient. $\mathrm{Pa}$ tients who require ovulation induction should fall into one of the following diagnostic categories.

\section{Patients with excess androgens including}

Polycystic Ovary Disease, Adrenal Androgenic

Hyperplasia, and combination of both

a. Patients with Polycystic Ovary Disease (PCO): The typical patient in this group has hirsutism and/or menstrual dysfunction. The ovaries are usually enlarged because they have numerous follicles partially stimulated and prepared for ovulation. Testosterone (T) is slightly elevated. FSH is slightly 
Table 1: Normal Range of Serum Levels of Hormones Needed in the Work-up of Anovulatory Patients. (Bowman Gray School of Medicine Laboratories*)

Hormones

Follicle-stimulating hormone (FSH)

Luteinizing hormone ( $\mathrm{LH})$

Thyroid stimulating hormone (TSH) Prolactin (PRL)

Testosterone " $\mathrm{T}$ "

Androstenedione "A"

Dehydroepiandrosterone sulphate

(DHEAS)

Serum Level

("Individual laboratories establish their own reference ranges.)

depressed and LH is slightly elevated with the ratio of LH:FSH being 2 or more. These patients usually have adequate endogenous estrogen and therefore they have withdrawal bleeding after progesterone or progestational therapy. A survey of the data presented in the literature shows that these patients respond adequately to clomiphene citrate with ovulation rates being $80 \%$ to $90 \%$ and pregnancy rates about $50 \%$ to $60 \% .^{1,2}$ Patients who do not respond to clomiphene citrate may be given dexamethasone $0.5 \mathrm{mg}$ daily at bed time in addition to the clomiphene citrate regimen. If this combined treatment fails to induce ovulation, then the human menopausal gonadotropin (HMG) "Pergonal", in combination with human chorionic gonadotropin (HCG) should be tried.

b. Patients with Adrenal Androgenic Hyperplasia $(\mathrm{AAH}):$ The adrenal hyperplasia in these patients is often due to a slight deficiency of 21-hydroxylase enzyme causing increased secretion of androgens, principally dehydroepiandrosterone (DHEA) and to a much lesser extent androstenedione (A). Both of these androgens can be metabolized into the more potent testosterone. These patients usually have hirsutism and/or menstual dysfunction and possible slight hypertrophy of the clitoris. Unlike levels in patients with PCO, and LH blood levels are normal or slightly decreased. T is slightly elevated but DHEA sulphate (DHEAS) is markedly elevated and can be suppressed by glucocorticoids. These patients may or may not respond to the progesterone withdrawal test, depending on the availability of the endogenous estrogen. Ovulation can be induced by the administration of low dose corticoids such as dexamethasone $0.5 \mathrm{mg}$ at bed time and $0.25 \mathrm{mg}$ in the morning. If this therapy fails to induce proper ovulation, addition of clomiphene citrate may solve the problem. In an anovulatory patient with adrenal involvement, suppression of the adrenals will decrease the production of DHEAS and A, causing a drop in circulating estrogens and androgens which allows a rise in gonadotropin secretion with subsequent follicular stimulation and growth. With the combined treatment of dexamethasone and clomiphene citrate, better results are achieved than with glucocorticoids alone. The combined treatment achieves ovulation and pregnancy rates of $84.5 \%$ and $46 \%$, respectively.' If this combined regimen fails to induce ovulation, HMG combined with HCG should be tried.

c. Patients with $\mathrm{PCO}$ and $\mathrm{AAH}$ : In these patients the ovaries as well as the adrenals are responsible for the excessive secretion of androgens. The signs and symptoms are similar to those seen in the previous two groups but the T and DHEAS serum levels should be more elevated. The diagnosis is made by comparing $\mathrm{T}$ and DHEAS serum levels before and after dexamethasone suppression test. While dexamethasone is being administered daily $0.5 \mathrm{mg}$ at bed time and $0.25 \mathrm{mg}$ in a.m. to induce ovulation, a blood sample is tested for T and DHEAS, 6 weeks after the dexamethasone therapy. The diagnosis is made when DHEAS is found to be suppressed because of its adrenal origin while $\mathrm{T}$ is either slightly suppressed or is unchanged indicating that it is of ovarian origin. Successful ovulation induction may be obtained by the combined therapy of dexamethasone and clomiphene citrate. If this therapy fails to induce ovulation, HMG and HCG should be tried.

\section{Patient with hypothalamic anovulation}

These patients admit to chronic stress; social, parental or marital. Clinically, the spectrum of amenorrhea ranges from mild to severe hypothalamic suppression. There is no rule of thumb covering laboratory tests for these patients. When the hypothalamus is mildly suppressed, serum gonadotropins are usually in the low normal range and the progesterone withdrawal bleeding test is positive indicting the presence of endogenous estrogen. Most of these patients bleed within 7 days after receiving 100-200 $\mathrm{mg}$ of progestrone in oil intramuscularly. These patients may respond to clomiphene citrate alone or combined with HCG. This combined treatment resulted in $67 \%$ ovulation and a $42 \%$ pregnancy rate. ${ }^{1}$ It is believed that the addition of HCG therapy compliments or replaces the LH surge which may be defective. A patient with severe hypothalamic suppression will have very low gonadotropins and probably no endogenous estrogen and therefore her progesterone withdrawal bleeding test will be negative. This type of patient will only respond to Pergonal (HMG-HCG) therapy.

\section{Postpill anovulation}

Anovulation manifested by amenorrhea after oral contraceptive use is reported to occur in fewer than $1 \%$ of pill users. ${ }^{3}$ The diagnosis is usually established 
after eliminating other endocrinopathies as a cause of the anovulation by taking a good history and ordering the pertinent laboratory tests most notably serum PRL, FSH and LH levels. It is believed that this type of anovulation is a self-limited condition, as more than $90 \%$ of these patients have normal menses within 6 to 12 months. For the exceptional patient who is anxious to become pregnant before spontaneous menses are resumed, we have found that the treatment of choice is clomiphene followed by HCG if there is no response to clomiphene therapy alone. ${ }^{1}$ Seventy percent of our patients ovulated with clomiphene therapy alone, another $10 \%$ ovulated after the addition of $\mathrm{HCG}$, and the remaining $20 \%$ had very low levels of endogenous estrogen and responded only to HMG-HCG therapy.

\section{Patients with amenorrhea-galactorrhea syndrome}

In a nonpregnant woman, galactorrhea with amenorrhea is a pathologic condition often caused by serious organic disease. However, emission of a few drops of clear, straw colored fluid during periareolar pressure should not be considered galactorrhea. The fluid must be milk colored for this diagnosis. The quantity of milky secretion or the involvement of one or both breasts is immaterial and any galactorrhea requies evaluation. When galactorrhea is associated with amenorrhea, the disorder is more serious. The galactorrhea-amenorrhea syndrome is believed to be mediated by hypothalamic factors. If the normal secretion of gonadotropin-releasing hormone $(\mathrm{GnRH})$ or prolactin inhibiting factor (PIF), or both, is interfered with, then amenorrhea, galactorrhea, or a combination, occurs because of unrestrained prolactin secretion by the pituitary, with minimal elaboration of FSH and $\mathrm{LH}$. The common causes of galactorrhea-amenorrhea syndrome are: 1) frequent breast manipulation, especially suckling; 2 ) medications such as phenothiazines, reserpine, opiates, amphetamines, or oral contraceptives; 3) primary hypothyroidism that can be explained by the increased activity of thyrotropin releasing hormone (TRH) stimulating prolactin secretion; 4) pituitary tumors that may be micro-or macro-adenomas.

Looking at factors involved in the galactorrheaamenorrhea syndrome, the importance of seeking a pituitary tumor is obvious. A careful history and thorough physical examination will rule out the first three factors. Since any degree of hyperprolactinemia may suggest the presence of a pituitary tumor, we recommend screening all women with amenorrheagalactorrhea syndrome for the magnitude of plasma prolactin elevation.

The treatment is obvious if the syndrome is caused by tranquilizers, breast manipulation, or hypothyroidism. If the syndrome is due to a pituitary micro-adenoma (less than $1 \mathrm{~cm}$ in diameter) or macro-adenoma, the treatment can be either medical or surgical. Serum PRL levels are usually high and must be suppressed to enable the hypothalamicpituitary axis to resume normal function. Microadenomas can be treated with bromocriptine, a dopamine agonist, to shrink the tumor and suppress PRL secretion. Therapy should be initiated at the lowest level, eg $1.25 \mathrm{mg}$. daily and titrated against serum prolactin levels until the latter fall to the normal range. Surgery is not indicated because these micro-adenomas tend to grow slowly and are usually well controlled by bromocriptine. Tumor function should be monitored regularly by checking serum PRL level every six months. If PRL levels increase sharply, a computed axial tomography (CT) scan of the sella is mandatory.

Pituitary macro-adenomas are better treated by transphenoidal microsurgery or proton-beam irradiation. A major side effect of irradiation is the insidious development of panhypopituitarism. Resolution of hyperprolactinemia may not occur after surgery, so most cases require postoperative treatment with bromocriptine. Some endocrinologists prefer to treat macro-adenomas with bromocriptine for one year. If size decreases, then surgery is not recommended and medical treatment is continued. The tumor function may be monitored in the same manner as that of micro-adenoma.

\section{Patient with proliferative phase defect (follicular maturation disorder)}

Women with proliferative phase defect are healthy and are usually infertile secondary to oligoovulation. The patients in this group share with those who had the mild hypothalamic disorder the long irregular cycles, but differ from them in that they usually ovulate, however not on a regular basis. Menstrual cycle length in patients with follicular phase defect are usually from 5 to 7 weeks. Sherman and Korenman ${ }^{4}$ studied a similar group of patients who had long, unpredictable intermenstrual intervals and found that the increased cycle length was always due to a prolonged follicular phase as measured by the time until the $\mathrm{LH}$ peak. In addition, they found that during the prolonged follicular phase, $\mathrm{LH}$ and FSH levels were within the range observed during the normal length cycles. Although normal fertility is the rule in these patients, it cannot be taken for granted, as for some patients with follicular phase defect, the menstrual cycles are not oligo-ovulatory but rather anovulatory. The ovarian follicles are suspected to be of poor quality causing prolongation of the proliferative phase with no actual ovulation. The importance of oligo-ovulation lies in the fact that it may be a precursor to amenorrhea or even premature ovarian failure and therefore patients in this category should be encouraged to complete their families at their earliest convenience. Clomiphene therapy is the treatment of choice for these patients as it induces 
regular ovulation in almost all of them. Clomiphene may play a role in increasing the number of ancillary follicles and in improving the growth pattern of the maturing follicles.

\section{Patients with luteal phase defect}

Luteal phase defect is defined as either a defect in progesterone output from the corpus luteum or an inadequate preparation of the endometrium. There is no definitive evidence in the literature that luteal phase inadequacy causes infertility and no conclusive proof that its correction enhances fertility. Luteal phase inadequacy is found in less than $5 \%$ of the unselected population. ${ }^{3}$ Tho, et al. ${ }^{6}$ showed that $25 \%$ of recurrent miscarriages had luteal phase inadequacy diagnosed by two endometrial biopsies. The patients who are at risk of developing luteal phase defects are: women who had repetitive early miscarriages, patients with hyperprolactinemia, patients using clompihene citrate, women undergoing strenuous athletic conditions, women who are over 35 years of age, patients with unexplained infertility and those with endometriosis. Patients secreting excess androgens may develop a luteal phase defect manifested by infertility or recurrent early miscarriages. Increased androgens can prolong the follicular phase and shorten the luteal phase. Androgens also increase the incidence of follicle atresia.

The diagnosis of a luteal phase defect is best made by endometrial biopsy. It is now generally agreed that progesterone levels, because of extreme variability, do not reflect corpus luteum function. The biopsy should be taken only from the fundus of the uterus and within a day or two of subsequent menses. The day of onset of the next menstrual period is called postovulatory day 14 . The pathologic specimen reading should correspond to the postovulatory day of biopsy counting backwards from the day menses started. A lag of greater than two days in two or more biopsied cycles is diagnostic. Basal body temperature (BBT) recordings do not have the quantitative ability to make the diagnosis of luteal phase inadequacy.

Patients with short luteal phase and those whose biopsies show glandular stromal asynchrony are better treated with clompihene citrate. These patients are thought to have low gonadotropins and poor follicular development which usually respond to clomiphene. On the other hand, for patients with glandular stromal synchrony in whom the dating of the endometrium lags by 2 days or more behind the expected date, particularly those in whom luteal phase length is normal, the treatment of choice is progesterone. The goal of progesterone supplementation is to replace inadequate progesterone output and to induce endometrial repair. Progesterone vaginal suppositories, $25 \mathrm{mg}$ twice daily is the recommended dose. The supositories must be give at 12 -hour inter- vals because progrestrone is rapidly absorbed and cleared from the body. Natural progesterone is the only form of progesterone which can be used to treat luteal phase defect. Progestogens like provera or norlutate are contraindicated in early pregnancy as they may interfere with implantation or even impede the function of the corpus luteum. Therapy begins on the third postovulatory day, as indicated by the BBT chart, and is continued until the period has begun, which will not usually be delayed more than one or two days. An endometrial biopsy in the first or second treatment cycle is imperative to identify patients in whom therapy is not working. Other causes of luteal phase defect should be treated by eliminating the etiologic factor. For example, hyperprolactinemia should be treated with bromocriptine.

\section{Kallmann's syndrome}

This syndrome is characterized by hypogonadotropic hypogonadism (primary amenorrhea), anosmia and color blindness. The syndrome is more common in males and the whole spectrum of clinical abnormalities may not be present in all cases. Ovulation can be induced by $\mathrm{GnRH}$ or HMG-HCG therapy.

\section{Ovulating agents:}

\section{Clomiphene citrate}

This is an orally active nonsteroidal agent distantly related to diethylstilbestrol. It is currently available in $50 \mathrm{mg}$ tablets under the name of Clomid or Serophene. It exerts only a very weak estrogenic effect, but its chemical similarity to estrogen is sufficient to achieve uptake and binding to estrogen receptors. Receiving only minimal estrogen signals because estrogen receptors are occupied by the clomiphene, the hypothalamus secretes more GnRH and the pituitary is stimulated to secrete large quantities of FSH and LH. As in the normal cycle, the rise in FSH stimulates a set of follicles to begin growth and maturation. Therefore, effectiveness of the drug is restricted to its ability to cause this FSH discharge.

The only indication for clomiphene therapy is infertility due to absent or infrequent ovulation. The proper candidates for this drug therapy are healthy individuals who have some evidence of pituitaryovarian activity as expressed by biologic levels of estrogen and a positive response to progestin withdrawal.

Clomiphene is given on the fifth day of the cycle and is administered for 5 days. The initial dose is 50 $\mathrm{mg} /$ day and if ovulation is not achieved in any cycle, the dose is increased in stepwise fashion by $50 \mathrm{mg}$ units to $150 \mathrm{mg} /$ day for 5 days. The BBT chart is essential in following the patient on clomiphene therapy. The BBT chart (Figures 1-3) provides evidence of ovulation and information about the ade- 
PREVIOUS CYCLES: LONGEST

JU- SHORTEST
THISCYCLE

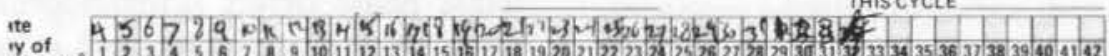

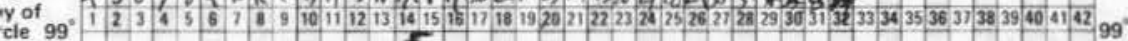

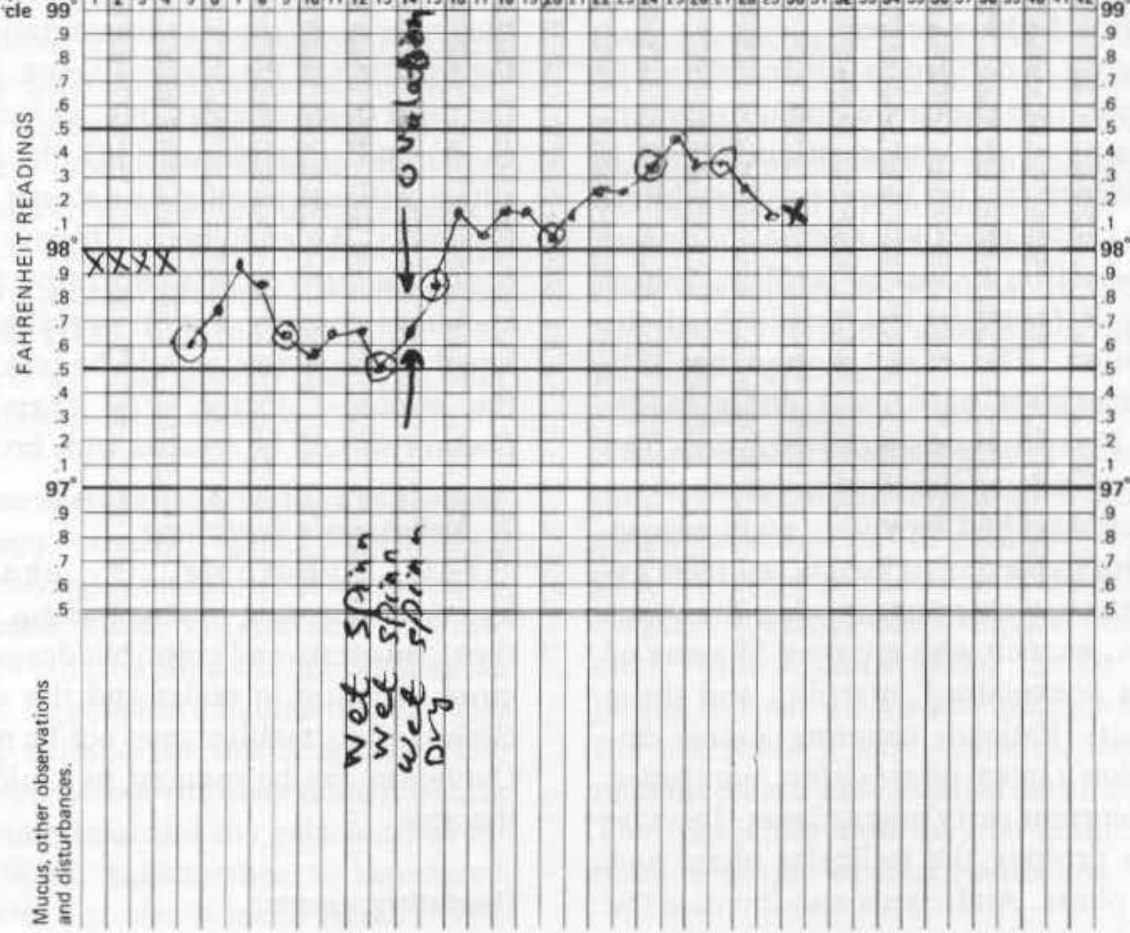

Figure 1 A normal biphasic basal body temperature chart. The arrows show the day of ovulation.

PREVIOUS CYCLES: LONGÉST
THOYSCYCLE

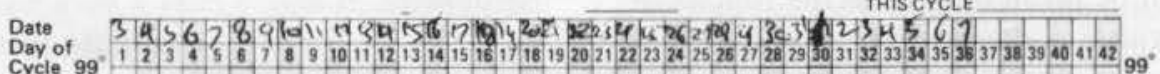

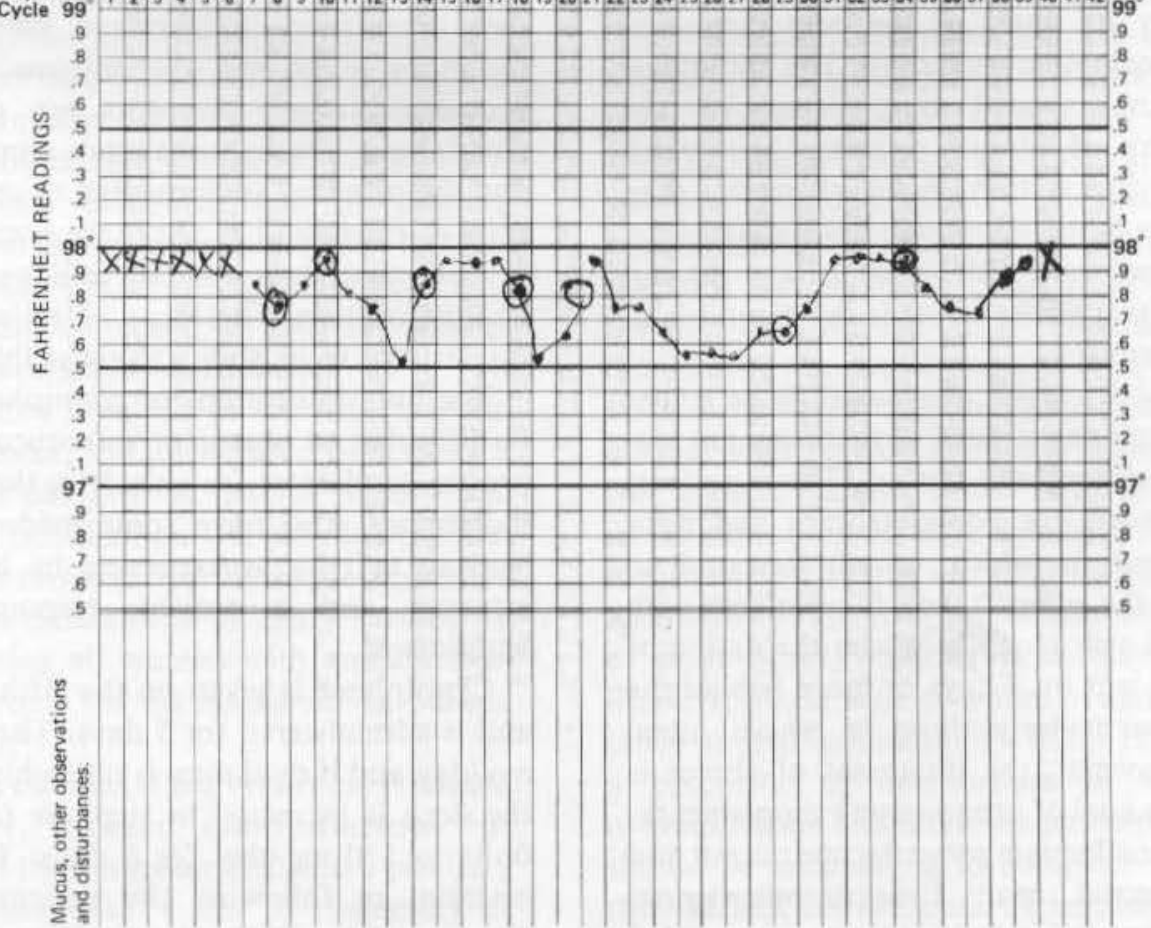

Figure 2 Anovulatory monophasic basal body temperature chart. 


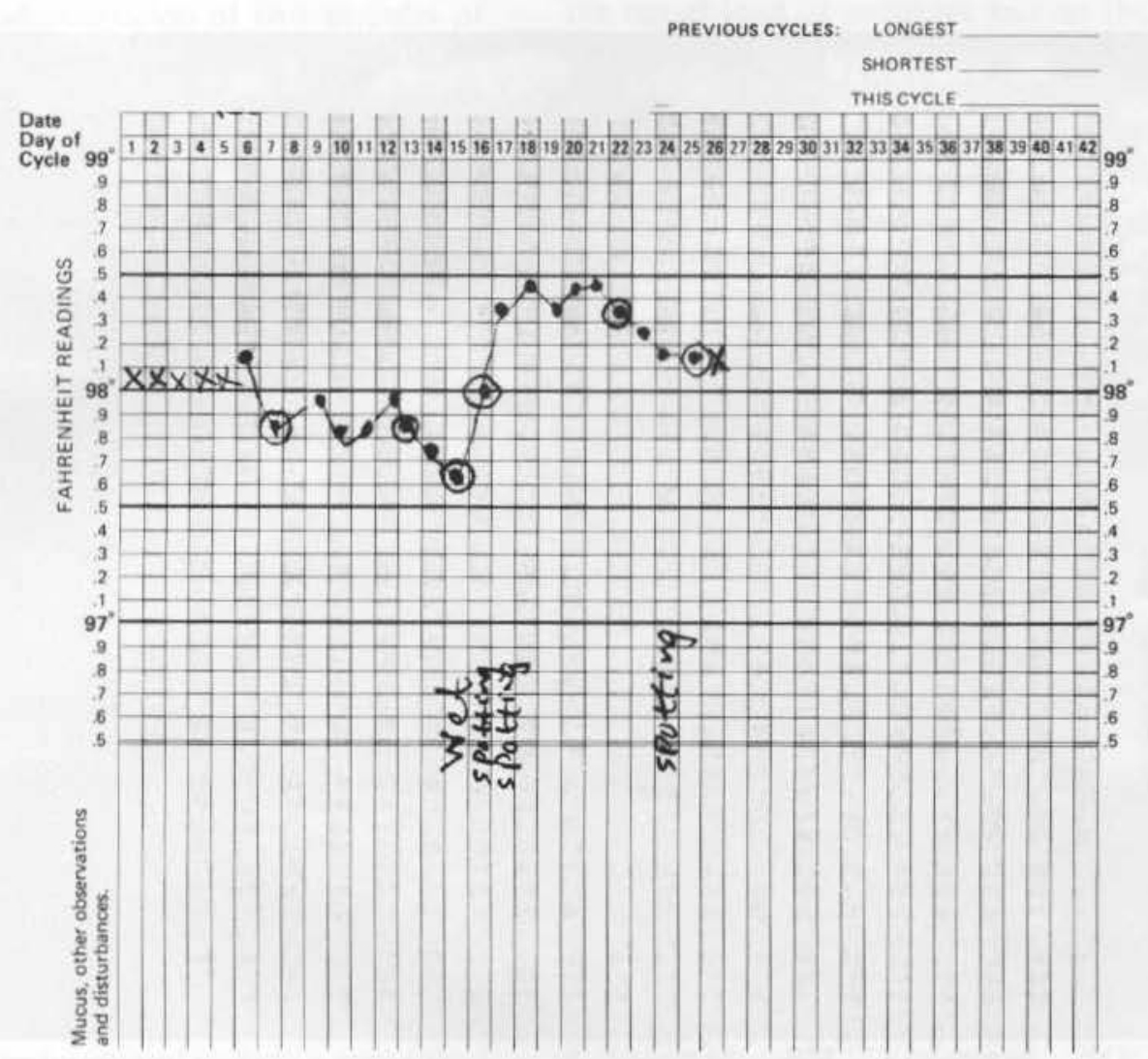

Figure 3 Basal body temperature chart shows short luteal phase. Ovulation took place on the 15th day of the cycle. Notice spotting days. Endometerial biopsy showed luteal phase defect.

quacy of the luteal phase since $75 \%$ of pregnancies occur in the first 6 cycles and only $15 \%$ occur at the $150 \mathrm{mg}$ level. Although clomiphene therapy should be withheld if there is any possibility of pregnancy, there has been no evidence that clomiphene is teratogenic in humans.

\section{Clomiphene and HCG}

This combination treatment is indicated when clomiphene alone fails to induce ovulation and when there is a short luteal phase (less than 12 days) manifested by the BBT chart. The rationale is to improve the mid-cycle LH surge. A single intramuscular dose of $10,000 \mathrm{IU}$ is given on the 7th to 10 th day after the last clomiphene tablet, when follicular maturation is at its peak as shown by serum estrogen level $(500-1,000 \mathrm{ng} / \mathrm{ml})$ and the size of the follicle as seen in a sonogram (18-22 $\mathrm{mm}$ in diameter).

\section{Glucocorticoids}

The excess androgens secreted by the adrenals or the ovaries may be reduced by the administration of glucocorticoids with consequent enhancement of pituitary gonadotropin release and improvement of ovarian follicular reactivity. ${ }^{7}$ When clomiphene fails to induce ovulation in a patient with excess an- drogens, the addition of dexamethasone usually solves the problem. We give dexamethasone 0.5 tablet daily at bed time till conception occurs; rarely $0.25 \mathrm{mg}$ in a.m. needs to be added.

\section{Pergonal (HMG)}

Pergonal is a purified preparation of gonadotropins extracted from the urine of postmenopausal women. Each ampule contains 75 IU of FSH and 75 IU of LH. We give two ampules IM daily starting day 3 of the menstrual cycle until follicular maturation is achieved. After 4 days of injections, the patient is brought back for sonographic visualization of the follicles (Figures 4 and 5), for blood levels of estrogen and for a mucus check. The frequency of the monitoring process will be adjusted as needed according to response, i.e. daily or alternate days. The dose, regimen and monitoring schedule is adjusted in subsquent cycles according to response and information obtained in initial cycles. When the mean follicle diameter (MFD) reaches 18-20 mm (16 $\pm \mathrm{mm}$ for PCO) and blood estrogen level is greater than $400 \mathrm{ng} / \mathrm{ml}$, a single IM injection of $10,000 \mathrm{IU}$ is given. Because of its structural and biologic similarity to $\mathrm{LH}, \mathrm{HCG}$ is used to simulate the mid-cycle LH ovulatory surge; ovulation occurs 


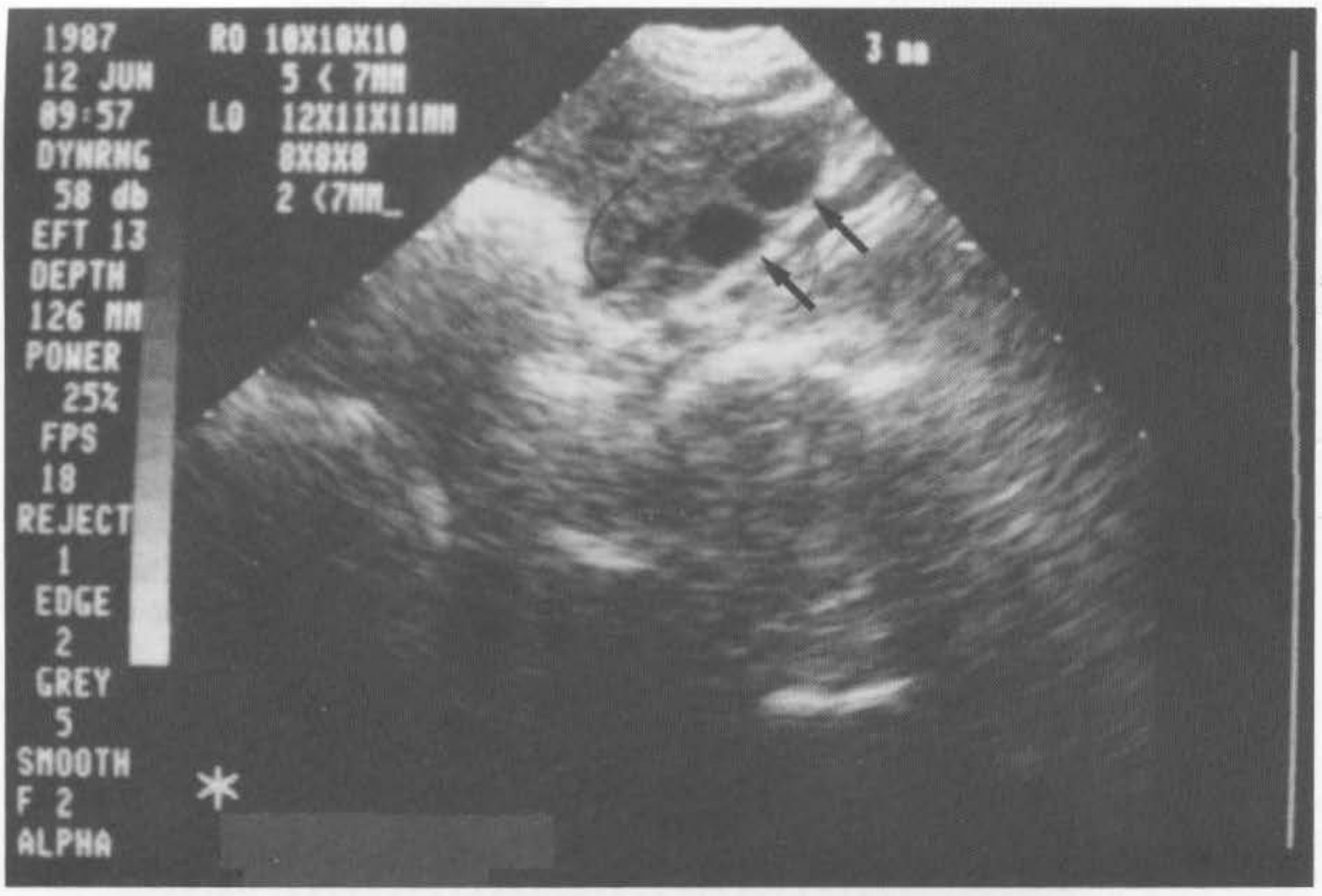

Figure 4 Ultrasound scan show two immature follicles measuring $12 \times 11 \times 11 \mathrm{~mm}$ each.

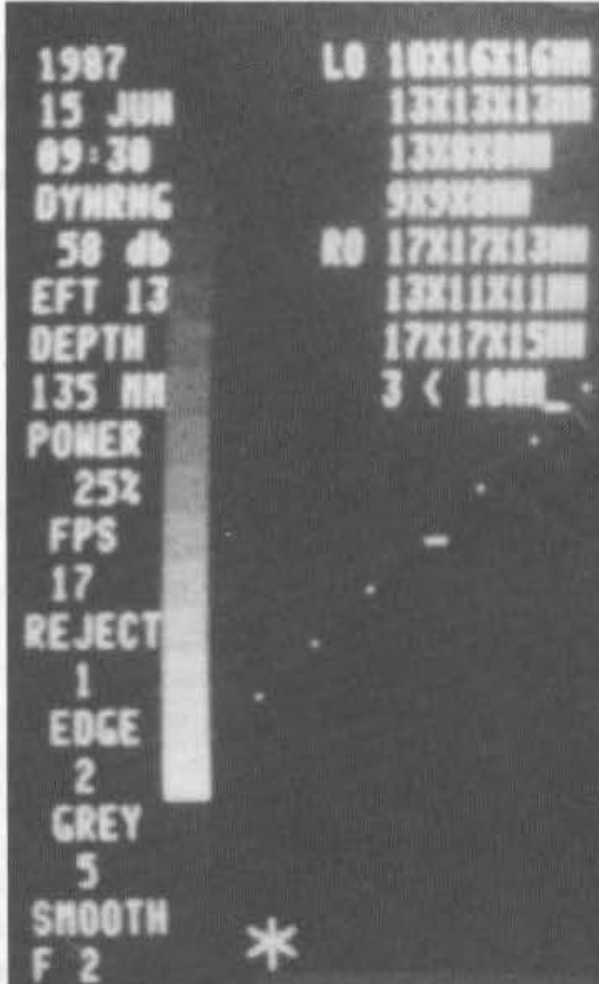

Figure 5 Ultrasound scan shows a mature follicle measuring $18 \times 16 \times 17 \mathrm{~mm}$. 
28-32 hours later. In most patients, ovulation will be achieved with the adminisration of two ampules of pergonal daily for 7 to 10 days. The patient is advised to have intercourse 24 hours after the HCG injection and if possible 12 hours later. Close observation and experience in the use of HMG are necessary to avoid complications such as hyperstimulation and multiple gestation. A reasonable trial of pergonal is considered to be approximately six ovulatory cycles. After this trial the histories and responses of the patient should be reviewed and a decision should be discussed with the couple to continue or discontinue therapy.

\section{Gonadotropin-releasing hormone (GnRH)}

This is a decapeptide hypothalamic hormone that controls the release of gonadotropins from the pituitary. Because of the very brief half-life of $\mathrm{GnRH}$, treatment has been given by multiple daily injections or by infusion. Several investigatiors have reported inducing ovulation in patients with hypogonadotropic hypognadism giving GnRH subcutaneously over a long period in pulsatile doses every 60 to 120 minutes using an infusion pump. The drug may be administered IV or IM intermittently. The pulsatile action simulates the physiologic GnRH secretion. ${ }^{8}$ Most recently the drug has been given by intranasal spray. The intranasal route is a convenient method for prolonged therapy, but larger doses of medication are required than when IM or IV administration is used. The theoretic advantages of pure GnRH administration are threefold: it is assumed to be more physiologic, there is less incidence of hyperstimulation syndrome and less incidence of multiple pregnancies. The drug produces no side effects, but patients are inconvenienced by wearing the pump.

\section{Bromocriptine mesylate (Parlodel)}

Bromocriptine is an ergot alkaloid that inhibits the pituitary secretion of prolactin. The drug has revolutionized treatment of patients with hyperprolactinemia manifested by amenorrhea, galactorrhea, or both. It is given as $1.25 \mathrm{mg}$ by mouth daily at bed time for two weeks, then the dose is increased to $2.5 \mathrm{mg}$ daily at bed time for another two weeks. The administration of this initial small dose is to avoid the side effects of nausea and vomiting. Later the dose is increased by $2.5 \mathrm{mg}$ increments depending on the initial level of prolactin and on the response to the treatment shown by the extent of the drop of the prolactin blood level. The usual dose is 5 to $10 \mathrm{mg}$ daily. Restoration of menstruation occurs in $90 \%$ of patients in two to three months. Ovulation is resumed in $80 \%$ and pregnancy occurs in $54 \%$ of recipients. ${ }^{9}$ Apparently there are no teratogenic effects when this therapy is taken early in pregnancy, but a positive pregnancy test mandates discontinuation of bromocriptine. There is no evidence that prolonged therapy achieves a permanent restoration of ovulatory menses. With discontinuation of the drug, amenorrhea recurs in the majority of patients.

\section{References}

1. Fayez, JA: Selection of patients for clomiphene citrate therapy. Obstet Gynecol 1976; 47:671.

2. Goldziehv, JW: Polycystic Ovarian Disease, Progress in Infertility. Edited by Behrman, S, Kistner, R. Boston: Little, Brown and Company, 1968:351.

3. Speroff, L, Glass, R, Kase, N: Clinical Gynecologic Endocrinology and Infertility. Baltimore: Williams and Wilkins Company, 1974:57.

4. Sherman, BM, Korenman, SG: Hormonal characteristics of the human menstrual cycle throughout reproductive life. J Clin Invest 1975;55:699.

5. Jones, GE, Pourmand, K: An evaluation of etiologic factors and therapy in 555 private patients with primary infertility. Fertil Steril 1962; 13:389.

6. Tho, PT, Byrd, JR, McDonough, PG: Etiologies and subsequent reproductive performance of 100 couples with abortion. Fertil Steril 1979; 32:398.

7. Chang, RJ, Abraham, GE: Effect of dexamethasone and clomiphene citrate. Fertil Steril 1976; 27:640.

8. Wong, PC, Ash, RH: The pulsatile administration of luteinizing hormone - releasing hormone for induction of ovulation. Int J Fertil 1986; 30:11.

9. Seppala, M, Hirvonen, E, Ranta, T: Bromocriptine treatment of secondary amenorrhea. Lancet 1976; 1:1154. 